\title{
Quantitative evaluation of yeast's requirement for glycerol formation in very high ethanol performance fed-batch process
}

\author{
Julien Pagliardini ${ }^{1}$, Georg Hubmann ${ }^{2,3}$, Carine Bideaux', Sandrine Alfenore', Elke Nevoigt ${ }^{2,3,4}$,
} Stéphane E Guillouet ${ }^{1^{*}}$

\begin{abstract}
Background: Glycerol is the major by-product accounting for up to 5\% of the carbon in Saccharomyces cerevisiae ethanolic fermentation. Decreasing glycerol formation may redirect part of the carbon toward ethanol production. However, abolishment of glycerol formation strongly affects yeast's robustness towards different types of stress occurring in an industrial process. In order to assess whether glycerol production can be reduced to a certain extent without jeopardising growth and stress tolerance, the yeast's capacity to synthesize glycerol was adjusted by fine-tuning the activity of the rate-controlling enzyme glycerol 3-phosphate dehydrogenase (GPDH). Two engineered strains whose specific GPDH activity was significantly reduced by two different degrees were comprehensively characterized in a previously developed Very High Ethanol Performance (VHEP) fed-batch process.

Results: The prototrophic strain CEN.PK113-7D was chosen for decreasing glycerol formation capacity. The finetuned reduction of specific GPDH activity was achieved by replacing the native GPD1 promoter in the yeast genome by previously generated well-characterized TEF promoter mutant versions in a gpd2 $\triangle$ background. Two TEF promoter mutant versions were selected for this study, resulting in a residual GPDH activity of 55 and $6 \%$, respectively. The corresponding strains were referred to here as TEFmut7 and TEFmut2. The genetic modifications were accompanied to a strong reduction in glycerol yield on glucose; the level of reduction compared to the wildtype was $61 \%$ in TEFmut7 and $88 \%$ in TEFmut2. The overall ethanol production yield on glucose was improved from $0.43 \mathrm{~g} \mathrm{~g}^{-1}$ in the wild type to $0.44 \mathrm{~g} \mathrm{~g}^{-1}$ measured in TEFmut7 and $0.45 \mathrm{~g} \mathrm{~g}^{-1}$ in TEFmut2. Although maximal growth rate in the engineered strains was reduced by 20 and 30\%, for TEFmut7 and TEFmut2 respectively, strains' ethanol stress robustness was hardly affected; i.e. values for final ethanol concentration $\left(117 \pm 4 \mathrm{~g} \mathrm{~L}^{-1}\right)$, growthinhibiting ethanol concentration $\left(87 \pm 3 \mathrm{~g} \mathrm{~L}^{-1}\right)$ and volumetric ethanol productivity $\left(2.1 \pm 0.15 \mathrm{~g} \mathrm{l}^{-1} \mathrm{~h}^{-1}\right)$ measured in wild-type remained virtually unchanged in the engineered strains.

Conclusions: This work demonstrates the power of fine-tuned pathway engineering, particularly when a compromise has to be found between high product yield on one hand and acceptable growth, productivity and stress resistance on the other hand. Under the conditions used in this study (VHEP fed-batch), the two strains with "fine-tuned" GPD1 expression in a gpd2 $\triangle$ background showed slightly better ethanol yield improvement than previously achieved with the single deletion strains gpd1 $\Delta$ or gpd2 $\Delta$. Although glycerol reduction is known to be even higher in a gpd $1 \Delta$ gpd $2 \Delta$ double deletion strain, our strains could much better cope with process stress as reflected by better growth and viability.
\end{abstract}

\footnotetext{
* Correspondence: stephane.guillouet@insa-toulouse.fr

'Université de Toulouse, INSA, UPS, INP, LISBP, 135 Av de Rangueil, F-31077 Toulouse France INRA, UMR792 Ingénierie des Systèmes Biologiques et des Procédés, F-31400 Toulouse, France CNRS, UMR5504, F-31400 Toulouse, France
} 


\section{Background}

Ideally, a micro-organism engineered for industrial biotechnology shows high product yield, final product concentration and productivity and can cope with process constraints. Achieving all these goals is a major challenge, particularly when it comes to modifications of the central carbon metabolism which is inherently coupled to energy and redox issues. Moreover, the cell's ability to cope with environmental stress can be severely affected. One prominent example for such a challenge is the reduction of glycerol formation in Saccharomyces cerevisiae (S. cerevisiae) in order to improve yield in ethanol production. Glycerol is produced from the glycolytic intermediate dihydroxyacetone phosphate (DHAP) which is reduced to glycerol-3-phosphate (G3P) by the two homologous isoenzymes of glycerol 3-phosphate dehydrogenase (GPDH), Gpd1 and Gpd2 [1,2]. G3P is then dephosphorylated into glycerol by the glycerol 3-phosphatases Gpp1 and Gpp2 [2,3]. In fact, glycerol is the main by-product beside carbon dioxide and biomass, accounting for up to $5 \%$ of the carbon [4].

A strain showing a reduced glycerol yield to the benefit of ethanol yield would result in substantial profit for the bioethanol industry. The challenge in reducing glycerol is that this compound and its formation fulfil major physiological functions in S. cerevisiae. Glycerol formation indeed participates in maintaining cytosolic redox balance [5-8] and in providing the intermediate G3P, essential for the biosynthesis of glycerophospholipids and triacylglycerols [9]. Glycerol is known to also contribute to stress management such as osmotic stress[10-12], heat, freezing/thawing or oxidative stress $[10,13]$.

Although glycerol formation by wild-type $S$. cerevisiae is, to a certain extent, strain dependent, it predominantly depends on the environmental conditions. The most important environmental factors are oxygen availability, type of nitrogen source, osmotic pressure, heat and $\mathrm{pH}$. For example, the presence of amino acids in the growth medium reduces the requirement of producing their carbon backbones. This results in a significantly lower generation of excess $\mathrm{NADH}$, i.e. lower glycerol formation when compared to minimal medium without any amino acids [5].

The first studies which aimed at redirecting the carbon flux toward ethanol by reducing glycerol synthesis focused on GPDH (see above). Mutants deleted in one or both isogenes encoding for GPDH were constructed in different strain backgrounds and tested for ethanolic fermentation [1,3,7,14-17]. Nissen et al [16] reported that single deletion mutants gpd $1 \Delta$ and gpd $2 \Delta$ showed respectively a $2.8 \%$ and a $4.7 \%$ ethanol yield improvement under anaerobic conditions and a $2.2 \%$ and $3.3 \%$ under aerobic conditions. The double deletion mutant was not able to grow under anaerobic conditions and showed a $12.7 \%$ yield improvement, but also a $29 \%$ reduction in biomass yield in aerobic conditions. Other metabolic engineering strategies have targeted redox metabolism with the goal to produce less net excess $\mathrm{NADH}$ during the biomass synthesis and organic acid formation [18]. Bro et al. [19] obtained a 3\% increase in the ethanol yield without any reduction in growth rate by by-passing the $\mathrm{NAD}^{+}$-dependent glycolytic conversion of glyceraldehyde to glycerate through the heterologous expression of a $\mathrm{NADP}^{+}$-dependent glyceraldehyde3-phosphate dehydrogenase. Nevertheless, these studies were made on low glucose concentration and did not imply industrially relevant process stresses such as high glucose and ethanol concentration. It was indeed shown that a $g p d 1 \Delta \operatorname{gpd} 2 \Delta$ double deletion mutant was severely affected in ethanol production (35\% decrease in final titre) and ethanol tolerance (25\% reduction in the $\mathrm{P}_{\text {criti- }}$ $\mathrm{cal} / \mu$ value (ethanol concentration at which growth stopped)) when placed under intensive ethanol production process[20].

Recent studies have combined single deletion of GPD1 or GPD2, engineering of redox metabolism and/or modification of yeast glycerol export [21-25] The best results, a $39.7 \%$ reduction in glycerol yield accompanied by a $12.3 \%$ ethanol yield improvement [25], were obtained with a strain deleted for GPD1, FPS1 and engineered for ammonium assimilation. Though, these results were obtained in a rich medium containing amino acids which is irrelevant in regards of most industrial processes. In addition, the use of rich medium compromises a correct conclusion about the redirection of carbon flow within these strains as long as catabolism of amino acids (in addition to glucose) is not taken into account.

Although some of the studies mentioned above gave interesting results, they suffer from a lack of information in terms of fermentative capacity in a high ethanol production process in which coping with process stress such as high ethanol titers becomes a critical issue for the yeast. For this purpose we previously developed a Very High Ethanol Performance fed-batch process [26] as a tool for studying the yeast physiology during ethanol production processes with high productivity and final ethanol concentration. We also showed that it was possible to reduce glycerol yield and final titer by finely monitoring the Respiratory Quotient (RQ) through glucose feeding in VHEP fed-batch [27]. However, this did not result in an increase in ethanol yield on glucose. We therefore searched for an avenue to further reduce glycerol formation in the VHEP fed-batch process. We envisaged identifying the optimal combination of process parameters and yeast strain genetic background for 
our process. However, it was clear from published data, that completely abolishing glycerol formation was accompanied by a drastic loss of process robustness $[16,20]$. We therefore envisaged an approach where glycerol formation capacity was strongly reduced but higher than in the gpd $1 \Delta$ gpd2 $\Delta$ double deletion strain. In this context, recent advances in yeast promoter engineering $[28,29]$ have opened new possibilities for finetuning of metabolic fluxes. Based on the knowledge about Gpd1 and Gpd2 activities in yeast and available promoters for fine-tuning gene expression, an appropriate engineering strategy was defined supported by metabolic flux calculations (see results). To engineer the according strains, GPD2 was deleted and GPD1 expression was reduced by replacing its native promoter by two previously constructed TEF1 promoter versions [29] with strongly reduced but different activities. We present here the kinetic analysis of the two genetically modified strains by characterizing (i) the effect of the genetic modification on product formation and growth (rates, yields and titers) and (ii) the robustness of the strains in our VHEP fed-batch process.

\section{Methods}

\section{Strains, media and growth conditions}

The Escherichia coli strain DH5 $\alpha^{\mathrm{TM}}$ (Invitrogen Corp., Carlsbad) was used for amplification of plasmids. The strain was grown in Luria-Bertani (LB) medium $(0.5 \%$ yeast extract, $1 \%$ peptone, $1 \% \mathrm{NaCl}, \mathrm{pH} 7)$ at $37^{\circ} \mathrm{C}$. $E$. coli transformation and isolation of plasmid DNA were carried out using standard techniques [30]. All Saccharomyces cerevisiae strains used in this study are listed in Table 1 and were derived from the prototrophic haploid wild-type strain CEN.PK 113-7D. For initial pre-cultivations, yeast strains were grown on YPD plates $\left(2 \mathrm{~g} \mathrm{~L}^{-1}\right.$ glucose, $1 \mathrm{~g} \mathrm{~L}^{-1}$ yeast extract, $1 \mathrm{~g} \mathrm{~L}^{-1}$ bacto peptone, $0.9 \mathrm{~g} \mathrm{~L}^{-1} \mathrm{NaCl}, 1.5 \mathrm{~g} \mathrm{~L}^{-1}$ agar) and stored in $30 \%$ glycerol at $-80^{\circ} \mathrm{C}$. All yeast strains used in this study are prototrophic allowing the use of minimum mineral media without any amino acid supplementation. All subsequent pre-cultures and fermentation experiments were carried out in synthetic mineral medium prepared as follows (all concentrations in $\mathrm{g} \mathrm{L}^{-1}$ ): $\mathrm{KH}_{2} \mathrm{PO}_{4}, 3.0$; $\left(\mathrm{NH}_{4}\right)_{2} \mathrm{SO}_{4}, 3.0 ; \mathrm{Na}_{2} \mathrm{HPO}_{4} 12 \mathrm{H}_{2} \mathrm{O}, 3.0$; sodium glutamate, 1.0; $\mathrm{MgSO}_{4} 7 \mathrm{H}_{2} \mathrm{O}, 0.5 ; \mathrm{ZnSO}_{4} 7 \mathrm{H}_{2} \mathrm{O}, 0.04$; $\mathrm{MnSO}_{4} \mathrm{H}_{2} \mathrm{O}, 0.0038 ; \mathrm{CoCl}_{2} 6 \mathrm{H}_{2} \mathrm{O}, 0.0005 ; \mathrm{CuSO} 4$
$5 \mathrm{H}_{2} \mathrm{O}, 0.0009 ; \mathrm{Na}_{2} \mathrm{MoSO}_{4} 2 \mathrm{H}_{2} \mathrm{O}, 0.00006 ; \mathrm{CaCl}_{2} 2 \mathrm{H}_{2} \mathrm{O}$, 0.023; $\left(\mathrm{NH}_{4}\right)_{2} \mathrm{Fe}\left(\mathrm{SO}_{4}\right)_{6} 6 \mathrm{H}_{2} \mathrm{O}, 0.023 ; \mathrm{H}_{3} \mathrm{BO}_{3}, 0.003$; pantothenate, 0.005 ; nicotinic acid, 0.005; meso-inositol, 0.125 ; thiamine, 0.005 ; pyridoxine, 0.005 ; para-aminobenzoic acid: 0.001, and biotin, 0.000012[31]. Three steps of propagation with increasing culture volumes ( 5 $\mathrm{mL}, 30 \mathrm{~mL}, 300 \mathrm{~mL}$ ) were carried out before inoculating the reactor for the VHEP fed-batch fermentations. Each pre-culture was grown for 12 hours and used as the inoculum for the next step at a $10 \% \mathrm{v} / \mathrm{v}$ ratio.

\section{Engineered yeast strain construction}

Genetic modifications of S. cerevisiae CEN.PK 113-7D carried out within this study comprise both the deletion of GPD2 and the replacement of native GPD1 promoter by two low-activity promoters (TEF1 promoters' versions see below). Gene deletion and promoter replacements based on homologous recombination in yeast were carried out according to the method described by Güldener et al. [32]. Transformation of S. cerevisiae was carried out according to Gietz et al. [33] using treatment with lithium acetate and polyethylene glycol. In order to allow expression of the antibiotic resistance genes cells directly after transformation were first incubated for at least $4 \mathrm{~h}$ at $30^{\circ} \mathrm{C}$ in YD containing $1 \%$ yeast extract and $1 \%$ glucose. Afterwards, cells were spread on YD agar plates supplemented with $7.5 \mu \mathrm{g} / \mathrm{ml}$ phleomycin (for GPD2 deletion) or $100 \mu \mathrm{g} / \mathrm{ml}$ geneticin G418 (for GPD1 deletion and integration of GPD1 promoter replacement cassettes).

GPD1 and GPD2 coding regions show strong similarities. In order to assure gene-specific homologous recombination of the GPD2 disruption cassette we used the GPD2 upstream region, which is different from the region upstream of GPD1. Primers used for amplification of the GPD2 disruption cassette and verification of correct gene disruption, listed in Table 2, were synthesized by Metabion International AG (Martinsried, Germany). Primers and PCR conditions used for the amplification of promoter replacement cassettes from our TEF1 promoter mutant collection were the same as previously described [29]. The thermostable $P f u$ DNA polymerase with proofreading activity was obtained from BIONEER (Korea) and used for amplification of both gene-disruption and promoter-replacement cassettes. Top DNA polymerase (BIONEER, Korea) was

Table 1 Saccharomyces cerevisiae strains used in this study

\begin{tabular}{|c|c|c|}
\hline Strain & Genotype & Source or reference \\
\hline CEN.PK 113-7D & Wild Type & van Dijken (2000) [52] \\
\hline TEFmut7* & 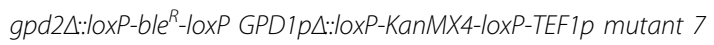 & This study \\
\hline TEFmut2* & gpd2A: IoxP-ble ${ }^{R}$-IoxP GPD1pA: IoxP-KanMX4-IoxP-TEF1p mutant 2 & This study \\
\hline
\end{tabular}

\footnotetext{
* These strains are isogenic to CEN.PK 113-7D.
} 
Table 2 Primers used for amplification the GPD2 disruption cassette and verification of its correct genomic integration

\begin{tabular}{|c|c|c|}
\hline No. & Function & Sequence \\
\hline P60* & $\begin{array}{l}\text { Forward primer for amplification of GPD2 deletion } \\
\text { cassette }\end{array}$ & 5'-TAGCTTACGGACCTATTGCCATTGTATTCCGATTA ATCTATTGTcagctgaagcttcgtacgc-3' \\
\hline P61* & $\begin{array}{l}\text { Reverse primer for amplification of GPD2 deletion } \\
\text { cassette }\end{array}$ & 5'-CACATTCTCACCTCTGGCTCGAAGATATGGGAATGCAATTCTGTgcataggccactagtggatctg-3' \\
\hline P62 & Forward primer for verification of GPD2 deletion & 5'-ACGATGG CTCTGCCATT-3' \\
\hline P63 & Reverse primer for verification of GPD2 deletion & 5'-GATCAGGATCGGCCACTA-3' \\
\hline
\end{tabular}

*Sequences in low chase letters correspond to the primer sequences for the amplification of the loxP-bleR-loxP deletion cassette from the plasmid pUG66 used as a template [32]. Nucleotides in capital letters are derived from S. cerevisiae S288c genome sequence for integration of the deletion cassette by homologous recombination at the GPD2 locus.

used in all diagnostic PCRs. PCR reaction mixtures were prepared according to the manufacturer's guidelines.

The GPD2 gene was deleted using the loxP-ble $e^{R}$ loxP cassette located on the plasmid pUG66 [32]. The gene $b l e^{R}$ confers resistance to phleomycin. The primers used for the amplification of the disruption cassette were P60 and P61 (Table 2). The GPD2 disruption cassette used here replaced 305 bp upstream of the GPD2 coding region and $360 \mathrm{bp}$ of the GPD2 coding sequence. The correct integration of the loxP-ble $e^{R}$-loxP cassette was verified by diagnostic PCR using the primer pair P62/ P63 (Table 2) and the following PCR conditions: $94^{\circ} \mathrm{C}$ for $1 \mathrm{~min}, 50^{\circ} \mathrm{C}$ for $1 \mathrm{~min}$, and $72^{\circ} \mathrm{C}$ for $2 \mathrm{~min}$. The PCR was performed in 30 cycles. If wild-type genomic DNA was used as a template, this diagnostic PCR resulted in a product of $855 \mathrm{bp}$ in size, whereas the product obtained from positive gpd $2 \Delta$ transformants had a size of $1.5 \mathrm{kbp}$.

In order to replace the native GPD1 promoter by promoters of much lower activities, the TEF1 promoter mutant versions 2 and 7 of our previously published promoter collection for fine-tuning gene expression in yeast [29] were used. The promoters were located on the described CEN/ARS plasmids p416-loxP-KmR-TEFmut2-yECitrine and p416-loxP-KmR-TEFmut7-yECitrine bearing the loxP-KanMX-loxP cassette upstream of the TEF1 promoter mutant 2 and 7, respectively. Integrations of the low-strength promoters were confirmed by PCR diagnosis using primers and PCR conditions as described earlier [29] except the temperature for primer annealing was set to $57^{\circ} \mathrm{C}$ instead of $60^{\circ} \mathrm{C}$. The PCR product obtained from native GPD1 promoter was 1.6 kbp, while a positive integration of the TEF1 promoter mutant 2 or 7 cassette yielded a product size of $2.6 \mathrm{kbp}$.

\section{Measurement of specific GPDH activity}

In order to determine the specific activity of glycerol 3phosphate dehydrogenase (GPDH), yeast strains were aerobically grown in shake flask cultures using the synthetic minimal medium as described above supplemented with $2 \%[\mathrm{w} / \mathrm{v}]$ glucose. The GPDH activity was measured in logarithmically growing cells (i.e. when $\mathrm{OD}_{600}$ reached about 1 ) according to a previously described method $[2,34]$.

\section{VHEP fed-batch protocol}

VHEP fed-batch fermentations were carried on in $5 \mathrm{~L}$ bioreactors B DCU B.BRAUN (SARTORIUS) with a starting volume of $3 \mathrm{~L}$. Temperature was set at $30^{\circ} \mathrm{C}$ and $\mathrm{pH}$ regulated at 4 by adding $14 \%$ ( $\mathrm{vol} / \mathrm{vol}) \mathrm{NH}_{3}$ solution. The reactor was flushed continuously with air; dissolved $\mathrm{O}_{2}$ was maintained above $20 \%$ of saturation by adapting the air flow and stirring rate in order to maintain fully aerated conditions. A sequential vitamin feeding strategy based on the growth profile [31] was applied. The fermentations were started with an initial glucose concentration of $100 \mathrm{~g} \mathrm{~L}^{-1}$. Whenever the residual glucose concentration was lower than $20 \mathrm{~g} \mathrm{~L}^{-1}$, glucose feeding was carried out with a $700 \mathrm{~g} \mathrm{~L}^{-1}$ glucose solution to restore a glucose concentration of $100 \mathrm{~g} \mathrm{~L}^{-1}$. At the later phase of fermentation, i.e.when the ethanol concentration was above $90 \mathrm{~g} \mathrm{~L}^{-1}$, the glucose feeding adjusted the concentration to $50 \mathrm{~g} \mathrm{~L}^{-1}$.

\section{Gas analysis}

Outlet and inlet gas analysis was performed using a mass spectrometer Proline Dycor ${ }^{2}$ a (Ametek Process Instrument). Gas analysis was performed on the outlet flow of the reactor every 5 minutes and on the inlet air every hour. The volumetric $\mathrm{O}_{2}$ consumption rate and the $\mathrm{CO}_{2}$ production rate were calculated from the differences between the inlet and outlet gas compositions, taking into account the evolution of the liquid volume in the reactor, the inlet airflow rate (regulated by a mass flowmeter), the temperature and the pressure.

\section{Analytical methods}

Yeast growth was evaluated by spectrophotometric measurements at $620 \mathrm{~nm}$ in a spectrophotometer Libra S4 (Biochrom) and calibrated against cell dry weight measurements. Cells were harvested by filtration on 0.45 $\mu \mathrm{m}$-pore-size polyamide membranes (Sartorius Biolab 
Product) and dried to a constant weight at $60^{\circ} \mathrm{C}$ under a partial vacuum $(200 \mathrm{~mm} \mathrm{Hg} \sim 26.7 \mathrm{kPa}$ ) for 24 hours. Rapid determination of glucose and ethanol concentrations from broth supernatants during fermentation was performed with an YSI analyser (YSI model 27 A; Yellow Springs Instruments).

Determination of ethanol, organic acids and glucose from supernatants was performed by HPLC using an Aminex HPX-87H+ column $(300 \mathrm{~mm} \times 7.8 \mathrm{~mm})$ and dual detection (refractometer and UV at $210 \mathrm{~nm}$ ) at $50^{\circ}$ C with $5 \mathrm{mM} \mathrm{H}_{2} \mathrm{SO}_{4}$ as an eluant (flow rate of $0.5 \mathrm{~mL}$ $\left.\min ^{-1}\right)$. Three independent metabolite quantifications (taking into account sampling, separation and HPLC quantification) showed that the measurement was reproducible; typically standard deviation was lower than $1 \%$ of mean value for ethanol and glucose and lower than $5 \%$ for glycerol and acetate.

\section{Chemicals}

All chemicals were of the highest analytical grade available.

\section{Determination of the cells' viability}

To determine cells' viability, the methylene blue technique was used as previously described [31].

\section{Assessment of ethanol evaporation}

When balancing aerobic ethanolic fermentation experiments, there is commonly a lack in carbon due to evaporation of ethanol. In fact, this may account for more than $20 \%$ of the total carbon $[16,35]$. To assess the part of evaporated ethanol, evaporation experiments were carried out under the same cultivation conditions as performed in this study but without yeast. The bioreactor was filled with 3 liters of the synthetic mineral medium used in this study and ethanol was added up to a concentration of $150 \mathrm{~g} \mathrm{~L}^{-1}$. Decrease in the ethanol concentration was recorded over time by taking regular liquid samples and HPLC quantification. The rate of evaporation was found to be dependent on ethanol concentration, aeration rate and liquid volume. No significant impact of stirring on evaporation was observed which is in accordance to a previous study [35]. Evaporation was investigated for the 2 aeration rates used during the fermentations $1 \mathrm{~L} \cdot \mathrm{mn}^{-1}$ and $0.5 \mathrm{~L} . \mathrm{mn}^{-1}$. Based on these data, an evaporation rate was attributed to each ethanol liquid concentration. Integration of this evaporation rate over time and liquid volume allowed calculating the evaporated ethanol in our yeast fermentations. The fact that the measured ethanol evaporation during the period of fermentation when cells had stopped producing ethanol exactly matched the calculated ethanol evaporation validated the method.

\section{Metabolic Flux Calculation}

Metabolic fluxes were calculated using a MFA based model extrapolated from a previously described Metabolic Descriptor $[27,36]$ The metabolic network was modified by adding mitochondrial compartmentation. Repartition of the reactions between cytosol and mitochondria was made according literature and previously described models [37-42]. Glycolysis, pentose phosphate pathway, synthesis of amino-acid precursors and nitrogen bases as well as formation of ethanol and glycerol were attributed to the cytosol. TCA cycle and respiratory chain were attributed to the mitochondria. Acetate and acetyl-coA synthesis were assumed to be localized in both cytosol and mitochondria. Segregation of amino acids synthesis between cytosol and mitochondria was inserted into the model according to the literature cited above. All transport reactions between the cytosol and the mitochondria were assumed to be simple transport reactions except for redox equivalent translocation via the glycerol shuttle. Our final metabolic network consists in 142 reactions, including 14 exchange reactions between the cell and external medium, 88 cytosolic reactions, 24 transport reactions between mitochondria and cytosol, and 16 mitochondrial reactions (cf. Additional file 1). Validity of the model was assessed using published data based on ${ }^{13} \mathrm{C}$ labelling experiments $[43,44]$.

The Yatp,x values and NADH balance were calculated from the results of metabolic flux calculation as follows:

$$
\begin{aligned}
& Y_{A T P, X}=\frac{\sum_{i} \alpha_{i}^{A T P O P} \times q_{i}^{G l y}+\sum_{i} \alpha_{i}^{A T P} \times q_{i}^{O P}}{N A D H_{\text {pro }}=} \sum_{i} \alpha_{i}^{N A D H} \times q_{i}^{G l y}+\sum_{i} \alpha_{i}^{N A D H} \times q_{i}^{T C A}+\sum_{i} \alpha_{i}^{N A D H} \times q_{i}^{A n a} \\
& N A D H_{c o n}=\alpha_{\text {Etoh }}^{\text {AdDH }} \times q_{\text {Etoh }}+\alpha_{\text {Glyce }}^{\text {NADH }} \times q_{\text {Glyce }}+\sum_{i} \alpha_{i}^{N A D H} \times q_{i}^{O P}
\end{aligned}
$$

$\alpha_{i}^{x}$ : Stoichiometric coefficient of metabolite $\mathrm{x}$ in reaction $\mathrm{i}$

$q_{i}^{y}$ : Rate of reaction i belonging to metabolic pathway $\mathrm{y}$

Ana: Anabolism

Gly: Glycolysis

$O P$ : Oxidative Phosphorylation

$T C A$ : Tricarboxylic acid cycle

Glyce: Glycerol

Etoh: Ethanol

NADHcon: NADH consumed

NADHpro: NADH produced 
The uncertainties of the TCA cycle fluxes values were estimated to impact the Yatp,x and NADH balance by less than $3 \%$.

\section{Results}

Design and construction of the GPD engineered strains supported by in silico flux calculations

The central question of this study was to evaluate whether and to what extent glycerol formation in S. cerevisiae can be reduced without severely affecting yeast's robustness, particularly ethanol tolerance under Very High Ethanol Performance fed-batch process conditions. Apart from the knowledge about the remarkably reduced ethanol tolerance of the gpd $1 \Delta$ gpd $2 \Delta$ double deletion strain, no quantitative data was available about the impact of reduced glycerol formation capacity on yeast's performance in our VHEP fed-batch process. A data set of specific substrate consumption and metabolite production rates measured during VHEP fed-batch cultivation with the CEN.PK 113-7D wild-type strain allowed us to calculate intracellular carbon fluxes including the one from DHAP to G3P necessary to fulfil biomass building block requirements. Flux calculations revealed that the ratio between the specific DHAP-toG3P conversion rate and the specific growth rate were constant over a long period of the growth. Based on our metabolic model, $5 \%$ of the total DHAP-to-G3P flux observed in the wild-type strain was required for anabolic reactions while $95 \%$ of this flux was diverted toward glycerol production (Figure 1). This 5\% flux should represent the minimum of carbon flux needed for anabolic requirements. Thus, one obligation for strain construction was to provide them with this minimal glycerol formation capacity.

In order to engineer $S$. cerevisiae strains with a strongly reduced but not completely abolished glycerol

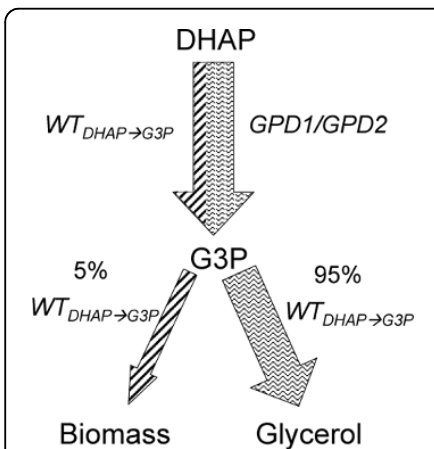

WT

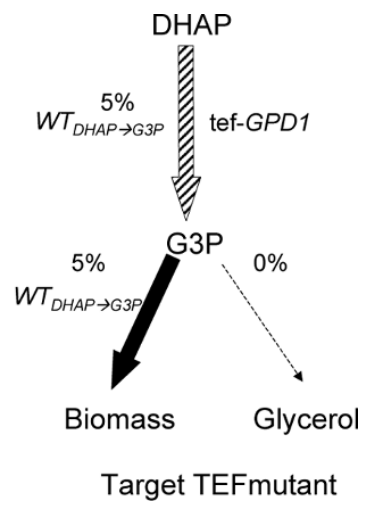

Figure 1 Flux repartition into the glycerol pathway. Fluxes were calculated using our Metabolic Descriptor model (see Methods, see Additional file 1) and experimental data obtained from a VHEP fedbatch fermentation with the wild type strain CEN.PK 113-7D. formation capacity, we envisaged to delete one isogene of GPDH and fine-tune the expression of the other one by replacing its native promoter by another one with much lower activity. We decided to delete GPD2 and fine-tune GPD1 expression since the latter is known to be responsible for the major part of GPDH activity and GPD2 deletion has been demonstrated to have no major impact on yeast physiology except when synthetic minimal medium was used under strictly anaerobic conditions [45]. In order to replace the native GPD1 promoter in CEN.PK 113-7D gpd2A background, two previously characterized mutated versions of the $S$. cerevisiae TEF1 were used (see Material and Methods). We chose the two weakest promoters available for this study, i.e. TEF1p mutant 2 (normalized promoter strength 7\%) and TEF1p mutant 7 (promoter strength $16 \%$ normalized to the native TEF promoter) [29]. Specific GPDH activity of the wild type was $0.041 \mathrm{U} / \mathrm{mg}$ protein. The down-regulation of GPD1 gene expression by GPD 1 promoter replacement in the gpd $2 \Delta$ background resulted in significantly reduced GPDH activities. The use of TEF1p mutant 7 upstream of GPD1 reduced GPDH activity to $55 \%(0.023 \mathrm{U} / \mathrm{mg}$ protein) whereas the use of TEF1p mutant 2 reduced GPDH activity to $6 \%(0.006 \mathrm{U} / \mathrm{mg}$ protein) compared to wildtype activity.

\section{Impact of reduced GPDH activity on fermentations parameters}

The two engineered strains TEFmut 7 and TEFmut 2 and the wild type were studied in VHEP fed-batch cultivation under comparable operating conditions in a synthetic mineral medium in order to precisely quantify the effect of the modulation of glycerol synthesis on ethanol production and growth capacities. Time courses of glucose consumption, biomass, ethanol and glycerol production are shown in Figure 2. All three fermentations showed two characteristic phases: a first "growth phase" where biomass was produced concomitant with ethanol, and a second "production phase", where growth had stopped due to ethanol inhibition but cells kept on producing ethanol. Growth of both the wild-type strain and the TEFmut7 strain ended after about 20 hours while growth of TEFmut 2 ended after 23 hours.

Calculated growth rate, biomass yield, glycerol yield as well as ethanol yield, final titer and productivity are summarized in Table 3. Carbon balances were closed to $89 \%$ for the wild type, to $94 \%$ for TEFmut 7 and to $94 \%$ for TEFmut2; the degree of reduction balances closed to $83 \%, 90 \%$ and $90 \%$, respectively. Evaluation of evaporated ethanol resulted in carbon and degree of reduction balances above $94 \%$ for all three fermentations. Acetaldehyde was not measured due to its volatility and could 


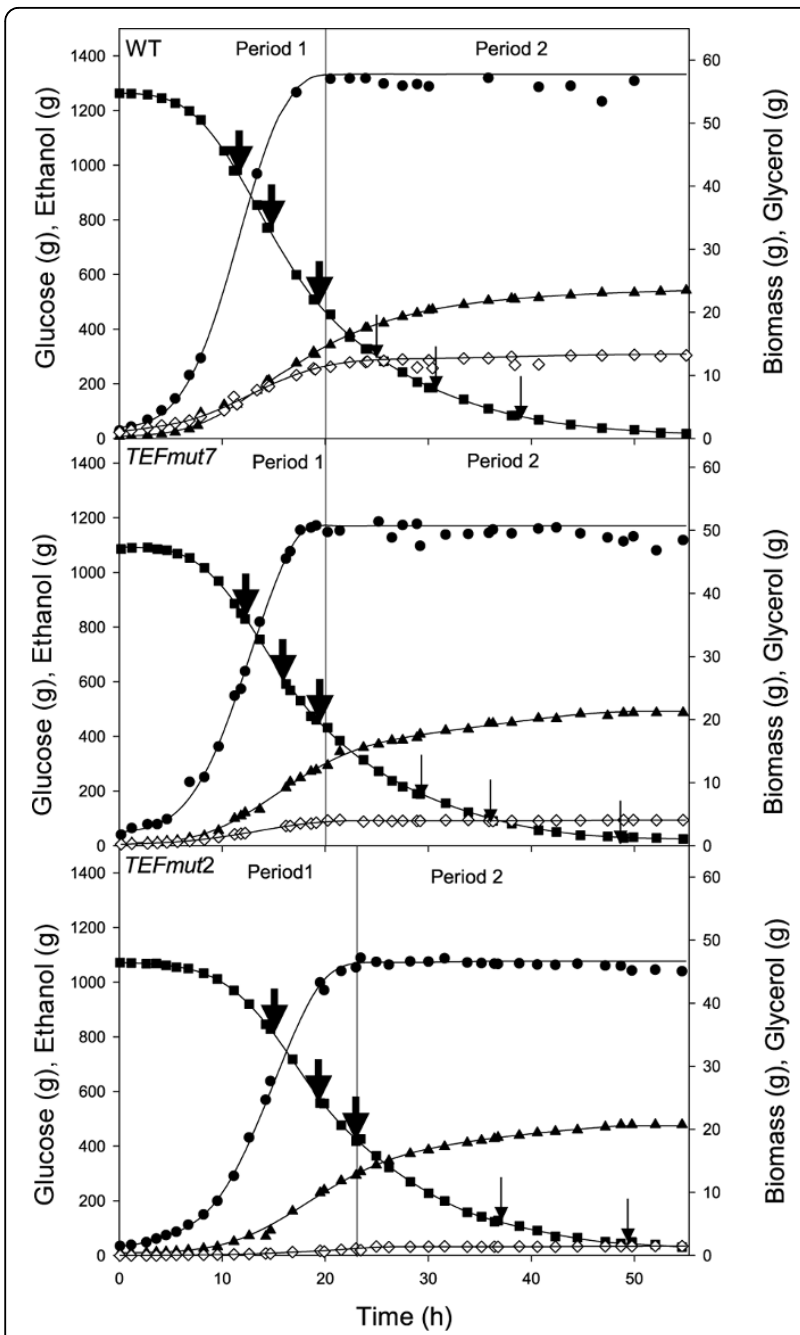

Figure 2 Substrate and products masses evolutions during the wild type and the mutants fermentations. Mass of glucose (black square), ethanol (black triangle), biomass (black circle) and glycerol (white diamond). Period 1 corresponds to the "growth/production phase" and period 2 to the "production phase". Thick arrows indicate the time points when glucose feeding was carried out in order to adjust glucose concentration in the fermenter up to $100 \mathrm{~g}$ $\mathrm{L}^{-1}$, thin arrows correspond to glucose feeding up to a concentration of $50 \mathrm{~g} \mathrm{~L}^{-1}$.

likely explain part of the deficit in carbon and degree of reduction balances.

The final biomass concentration obtained for the wildtype strain was $15.7 \mathrm{~g} \mathrm{~L}^{-1}$ while both modified strains showed a final biomass concentration of $14.5 \mathrm{~g} \mathrm{~L}^{-1}$. Final glycerol concentration was $3.1 \mathrm{~g} \mathrm{~L}^{-1}$ for the wildtype strain compared to $1 \mathrm{~g} \mathrm{~L}^{-1}$ for TEFmut7 and $0.4 \mathrm{~g}$ $\mathrm{L}^{-1}$ for TEFmut2. The final ethanol concentration reached was $117 \mathrm{~g} \mathrm{~L}^{-1}$ for the wild-type and slightly reduced to $112 \mathrm{~g} \mathrm{~L}^{-1}$ for TEFmut7 and $114 \mathrm{~g} \mathrm{~L}^{-1}$ for TEFmut2. At the end of fermentation, acetate concentration reached $5.5 \mathrm{~g} \mathrm{~L}^{-1}$ in the wild type and $5.1 \mathrm{~g} \mathrm{~L}^{-1}$ in the two mutants.

\section{Impact of reduced GPDH activity on fermentation kinetic parameters}

The reduction of GPDH activity in the engineered strains led to a decrease in the maximum specific glycerol production rate from $0.083 \mathrm{~g} \mathrm{~g}_{\mathrm{DCW}} \mathrm{h}^{-1}$ in the wild type to $0.023 \mathrm{~g} \mathrm{~g}_{\mathrm{DCW}} \mathrm{h}^{-1}$ and $0.004 \mathrm{~g} \mathrm{~g}_{\mathrm{DCW}} \mathrm{h}^{-1}$ in TEFmut7and TEFmut2, respectively. This corresponds to 28 and $5 \%$ residual rates in TEFmut7and TEFmut 2 compared to the wild type, respectively. However, the maximum specific growth rate and the maximum specific ethanol production rates were also reduced in the strains engineered for lower GPDH activity. The $\mu_{\max }$ was $0.35 \mathrm{~h}^{-1}$ for the wild type, $0.28 \mathrm{~h}^{-1}$ for TEFmut7 and $0.24 \mathrm{~h}^{-1}$ for TEFmut2. The maximum specific ethanol production rates were $1.31 \mathrm{~g}_{\text {ethanol }} \mathrm{g}_{\mathrm{DCW}}{ }^{-1} \mathrm{~h}^{-1}$ in the wild type, $1.10 \mathrm{~g}_{\text {ethanol }} \mathrm{g}_{\mathrm{DCW}}{ }^{-1} \mathrm{~h}^{-1}$ in TEFmut7 and 0.98 gethanol $\mathrm{g}_{\mathrm{DCW}}{ }^{-1} \mathrm{~h}^{-1}$ in TEFmut2 corresponding to $16 \%$ and $25 \%$ reduction in the strains TEFmut 7 and TEF$m u t 2$, respectively. Nevertheless, the overall volumetric ethanol productivity was hardly affected in TEFmut7 and TEFmut2, i.e. $2.2 \pm 0.1$ and $2.3 \pm 0.1 \mathrm{~g} \mathrm{~L}^{-1} \mathrm{~h}^{-1}$, respectively compared to $2.1 \pm 0.15 \mathrm{~g} \mathrm{~L}^{-1} \mathrm{~h}^{-1}$ for the wild type.

\section{Impact of reduced GPDH activity on yields}

Glycerol yield based on consumed glucose was $0.0051 \mathrm{~g}$ $\mathrm{g}^{-1}$ and $0.0016 \mathrm{~g} \mathrm{~g}^{-1}$ for TEFmut7 and TEFmut2, respectively, corresponding to $39 \%$ in TEFmut 7 and $12 \%$ in TEFmut2 compared to the wild type strain $\left(0.013 \mathrm{~g} \mathrm{~g}^{-1}\right)$. The glycerol yields per $g$ of biomass during the growth phase were also much lower for TEFmut7 and TEFmut2 (i.e. 0.06 and $0.02 \mathrm{~g} \mathrm{~g}^{-1} \mathrm{DCW}$ ) respectively, compared to $0.14 \mathrm{~g} \mathrm{~g}^{-1} \mathrm{DCW}$ for the wild type.

The biomass yields on glucose in TEFmut7 and TEFmut2 were slightly lower in the strains with the reduced GPDH activity, i.e. $0.091 \mathrm{~g}_{\mathrm{DCW}} \mathrm{g}_{\mathrm{glucose}}{ }^{-1}$ for TEFmut7 and $0.087 \mathrm{~g}_{\mathrm{DCW}} \mathrm{g}_{\text {glucose }}{ }^{-1}$ TEFmut 2 compared to 0.093 $\mathrm{g}_{\mathrm{DCW}} \mathrm{g}_{\text {glucose }}{ }^{-1}$ for the wild type. The overall acetate yields based on consumed glucose were similar for all three strains and reached $0.017 \mathrm{~g} \mathrm{~g}^{-1}$.

As a result, the overall ethanol production yield on glucose was increased in the two engineered strains. While the wild-type strain produced $0.43 \mathrm{~g} \mathrm{~g}^{-1}, T E F$ mut7 formed $0.44 \mathrm{~g} \mathrm{~g}^{-1}$ and TEFmut2 $0.45 \mathrm{~g} \mathrm{~g}^{-1}$. As visible from Table 3 , the strain differences in the overall ethanol yields were solely due to the differences observed during the growth phase. In this phase, ethanol yields reached $0.41 \mathrm{~g} \mathrm{~g}^{-1}$ for the wild type, $0.42 \mathrm{~g} \mathrm{~g}^{-1}$ for TEFmut7 and $0.44 \mathrm{~g} \mathrm{~g}^{-1}$ for TEFmut2. During the "production phase", no significant variation of ethanol yield 
Table 3 Fermentation characteristics for S. cerevisiae wild-type strain CEN.PK 113-7D and the two mutants

\begin{tabular}{|c|c|c|c|}
\hline & WT & TEFmut7 & TEFmut2 \\
\hline Fermentation Time (h) & 55 & 49 & 49 \\
\hline Growth Time (h) & 20 & 20 & 23 \\
\hline Final Volume (L) & 3.10 & 2.89 & 2.92 \\
\hline$\mu_{\max }\left(h^{-1}\right)$ & $0.35 \pm 0.02$ & $0.28 \pm 0.02$ & $0.24 \pm 0.02$ \\
\hline DCWmax $\left(g^{-1}\right)$ & $15.7 \pm 0.5$ & $14.5 \pm 0.5$ & $14.5 \pm 0.5$ \\
\hline YDCW/glucose $\mathbf{g ~ g}^{-1}$ & $0.093 \pm 0.006[5-15 h]$ & $0.091 \pm 0.007[5-15 h]$ & $0.087 \pm 0.005[5-17 \mathrm{~h}]$ \\
\hline Yethanol/glucose $\mathrm{g} \mathrm{g}^{-1}$ (growth) & $0.41 \pm 0.006[0-20 \mathrm{~h}]$ & $0.42 \pm 0.01[0-20 \mathrm{~h}]$ & $0.44 \pm 0.005[0-23 \mathrm{~h}]$ \\
\hline Yethanol/glucose $\mathrm{g} \mathrm{g}^{-1}$ (overall) & $0.43 \pm 0.005[0-55 h]$ & $0.44 \pm 0.008[0-49 h]$ & $0.45 \pm 0.003[0-49 h]$ \\
\hline Y glycerol/glucose $\mathrm{mg} \mathrm{g}^{-1}$ & $13 \pm 0.01[0-20 \mathrm{~h}]$ & $5.1 \pm 0.2[0-20 \mathrm{~h}]$ & $1.6 \pm 0.1[0-23 \mathrm{~h}]$ \\
\hline [ethanol] final $\left(\mathrm{g} \mathrm{L}^{-1}\right)$ & $117 \pm 4$ & $112 \pm 4$ & $114 \pm 4$ \\
\hline [glycerol] final $\left(\mathrm{g} \mathrm{L}^{-1}\right)$ & $3.1 \pm 0.1$ & $1 \pm 0.1$ & $0.4 \pm 0.1$ \\
\hline Ethanol Productivity $\left(\mathrm{g} \mathrm{L}^{-1} \mathrm{~h}^{-1}\right)$ & $2.13 \pm 0.15$ & $2.2 \pm 0.15$ & $2.3 \pm 0.15$ \\
\hline Pcritical $/ \mu\left(\mathrm{g} \mathrm{L}^{-1}\right)$ & $87 \pm 3$ & $85 \pm 3$ & $86 \pm 3$ \\
\hline
\end{tabular}

$\mu_{\text {max }}:$ maximum specific growth rate; $\mathrm{DCW}_{\text {max }}$ maximum cell concentration; Yi/glucose: production yield of the constituent $\mathrm{i}$ on glucose; Pcritical/ $\mu$ : ethanol concentration at which growth stopped; "growth phase": fermentation phase of yeast growth; "production phase": fermentation phase after yeast growth stopped but ethanol production continued.

could be observed between the wild type and the two engineered strains $\left(0.47 \mathrm{~g} \mathrm{~g}^{-1}\right)$.

We also calculated oxygen to biomass yield for all three strains. The average oxygen to biomass yield during the growth phase of the wild type was $6 \mathrm{mmol}_{\mathrm{O} 2}$ $\mathrm{g}_{\mathrm{DCW}}{ }^{-1}$. While there was no significant difference compared to TEFmut7 $\left(7 \mathrm{mmol}_{\mathrm{O} 2} \mathrm{~g}_{\mathrm{DCW}}{ }^{-1}\right)$, the strain TEFmut 2 showed a significantly higher yield of $10 \mathrm{mmol}_{\mathrm{O} 2}$ $\mathrm{g}_{\mathrm{DCW}}{ }^{-1}$.

\section{Impact of reduced GPDH activity on ethanol tolerance}

Figure 3 shows the evolution of both the specific growth and ethanol production rates as a function of the actual ethanol concentration in the bioreactor for each strain. The ethanol concentration at which uncoupling between growth and ethanol production occurs is usually referred to as Pcritical $/ \mu$. This parameter characterizes the strain-dependent growth inhibition by ethanol. Pcritical $/ \mu$ was about $87 \mathrm{~g} \mathrm{~L}^{-1}$ for the wild type, $85 \mathrm{~g} \mathrm{~L}^{-1}$ and $86 \mathrm{~g} \mathrm{~L}^{-1}$ for TEFmut7 and TEFmut2, respectively. Evaluation of cell viability by the methylene blue method indicated that ethanol tolerance of the GPDengineered strains was only slightly reduced compared to the wild type (Figure 4).

\section{Comparative flux analysis of the strains}

In order to better understand the metabolic reorganization upon the modulation of the glycerol synthesis pathway at the cell level, metabolic flux calculations were carried out for each strain. However in order to take into account the differences in $\mu_{\max }$ between the strains, the experimentally obtained specific consumption and production rates were chosen at $\mu_{\max }$ and normalized to a biomass production rate of $1 \mathrm{~g} \mathrm{~g}^{-1} \mathrm{~h}^{-1}$. The results of this calculation are reported in Figure 5. It shows that the DHAP-to-G3P flux at $\mu_{\max }$ was only $39 \%$ and $11 \%$ in TEFmut7 and TEFmut2, respectively compared to the level observed in the wild type. Moreover flux calculation also indicated that the modulation of the glycerol pathway led to a global metabolic reorganization pointed

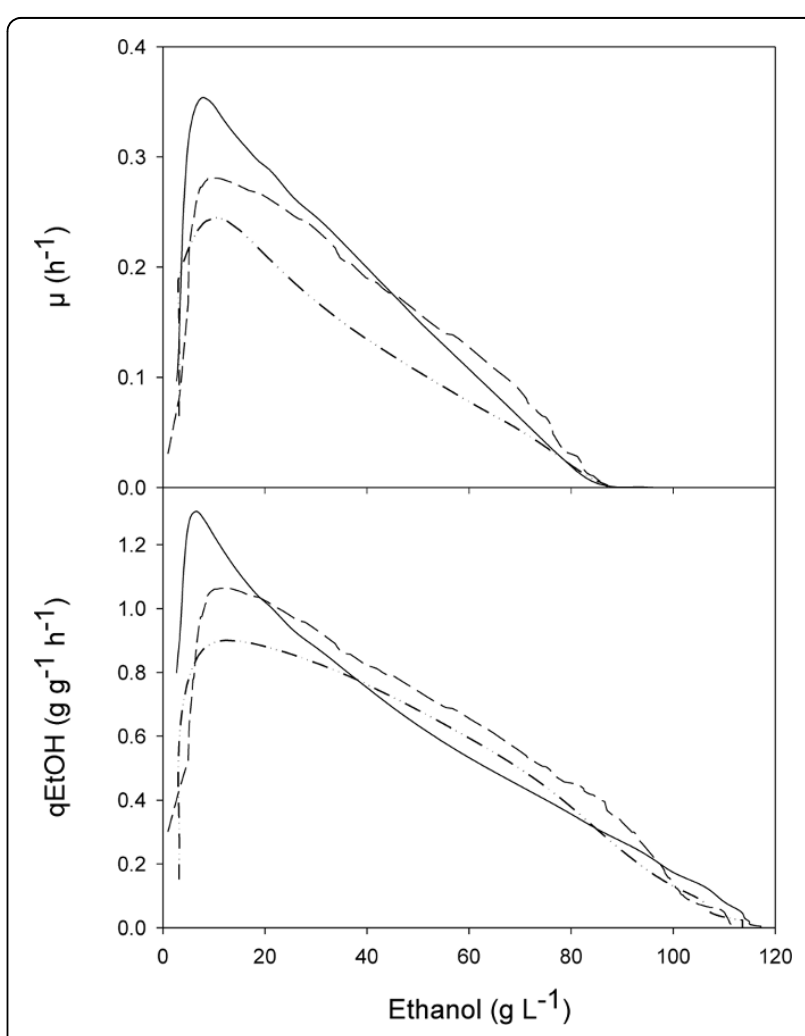

Figure 3 Specific growth rates $(\mu)$ and specific ethanol production rate (qEtOH) as functions of ethanol concentration. Strains: wild type CEN.PK 113-7D ( ${ }^{\prime}$ ), TEFmut7 (- - -), TEFmut2. (- ..). 


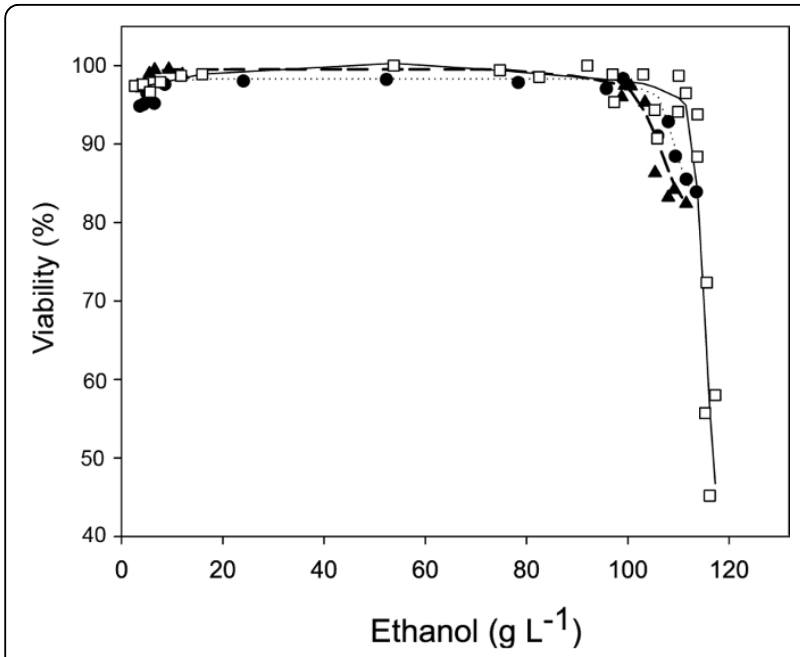

Figure 4 Cell viability as a function of ethanol concentration. Strains: wild type CEN.PK 113-7D (white square), TEFmut7 (black triangle), TEFmut2 (black circle).

out by the increased normalized rates of ethanol production, glycolysis, NADH mitochondrial shuttles and respiration.

\section{Discussion}

Aiming at determining to what extent glycerol formation may be reduced without affecting drastically the strain robustness in a VHEP fed-batch process, the modulation of glycerol synthesis capacity of $S$. cerevisiae was obtained by replacing the native promoter of GPD1 with promoters of significantly lower activities in a CEN.PK113-7D gpd2A background resulting in the strains TEFmut7 and TEFmut2.

\section{Fine-tuning of the glycerol synthesis pathway led to improved ethanol yield}

The two engineered strains led to a reduction of glycerol yield on glucose by $61 \%$ for TEFmut 7 and $88 \%$ in TEFmut 2 compared to the wild type strain. The metabolic flux calculation from the experimental data set of TEFmut7 and TEFmut2, respectively, showed that the DHAP-to-G3P flux was evaluated at 39 and $11 \%$ of the one calculated in the wild type (Figure 5). The reduction of the glycerol production in the two engineered strains was accompanied by a slightly increased ethanol yield on glucose (2.3\% for TEFmut7 and $4.6 \%$ for TEFmut 2 ). For comparison, in aerobic conditions, simple deletion mutants gpd $1 \Delta$ and $\operatorname{gpd} 2 \Delta$ of TN1 strain showed respectively a $2.2 \%$ and $3.3 \%$ yield improvement, whereas double deletion gpd1 1 gpd $2 \Delta$ strain showed a $12 \%$ improvement $\left(0.39 \mathrm{~g} \mathrm{~g}^{-1}\right)$ [16]. A $10 \%$ ethanol yield improvement was also obtained in a double deletion gpd $1 \Delta$ gpd $2 \Delta$ strain of W303-1A but was accompanied by a drastic loss of robustness towards ethanol stress [20].

In terms of carbon balance (Figure 6), the reduction of the glycerol production could not completely explain the gain in the ethanol yield. Obviously, a decrease in biomass production also contributed to this improvement. Metabolic flux calculations pointed out a decrease in the ATP-to-biomass yield (Yx,ATP) concomitantly with the decrease in the biomass production yield. The $\mathrm{Yx}$, ATP value was 9.7, 9.1 and $7.8 \mathrm{~g}_{\mathrm{DCW}} \mathrm{mol}_{\mathrm{ATP}}{ }^{-1}$, in the wt, the TEFmut7 and the TEFmut2, respectively. A decrease in the ATP yield related to the decrease in glycerol production was already observed in RQ controlled fermentation experiment [27]. This diminution of the biomass and ATP yield could be linked to the deficit of cytosolic NADH oxidation (discussion see below) and to the stress management. The cell stress management may cost more energy to the mutant strains under VHEP conditions due to high glucose and ethanol concentrations and high osmolarity (rise from 0.7 to $2.9 \mathrm{Osm} \mathrm{kg}^{-1}$ during the whole cultivation). For transport systems energetically related to the proton gradient, the cost in "equivalent" ATP may be higher in the strains producing less glycerol, known as the main compatible solute in yeast. For instance, osmoregulatory mechanisms such as $\mathrm{K}^{+}$homeostasis, glycerol exporter (FPS1) and compatible solute synthesis requiring appreciable ATP turnover [46] may be more requested in strains impaired in glycerol formation. Metabolic flux calculation showed that the mutant strains generated more energy than the wt to produce a similar amount of biomass (Figure 5) through a higher flux in the central carbon metabolism. Higher maintenance coefficients and higher TCA cycle flux were already reported in the literature during osmoregulation in S. cerevisiae [46,47].

Fine-tuning of the glycerol synthesis pathway altered the growth rate but not the ethanol tolerance in VHEP

The reduction of the glycerol formation in the two mutant strains led to a concomitant decrease in the maximal specific growth rate $\left(\mu_{\max }\right), 20 \%$ and $31 \%$ lower in the strains TEFmut7 and TEFmut2, respectively, comparatively to the wt strain. Nevertheless, based on the metabolic flux calculation, the DHAP-to-G3P rate should have been high enough for both strains to sustain the $\mu_{\max }$ of the wild type. Similarly a $55 \%$ decrease in the $\mu_{\max }$ was reported in gpd1 $1 \Delta$ gpd $2 \Delta$ strain under aerobic conditions, explained by the limited ability to reoxidize $\mathrm{NADH}$ to $\mathrm{NAD}^{+}$in the cytosolic compartment [16]. The deficit of cytosolic NADH oxidation due to the decrease in glycerol formation capacity could be partly compensated by the increase in the oxygen consumption through the respiration, observed in the mutant strains. Figure 7 indeed shows the increased 


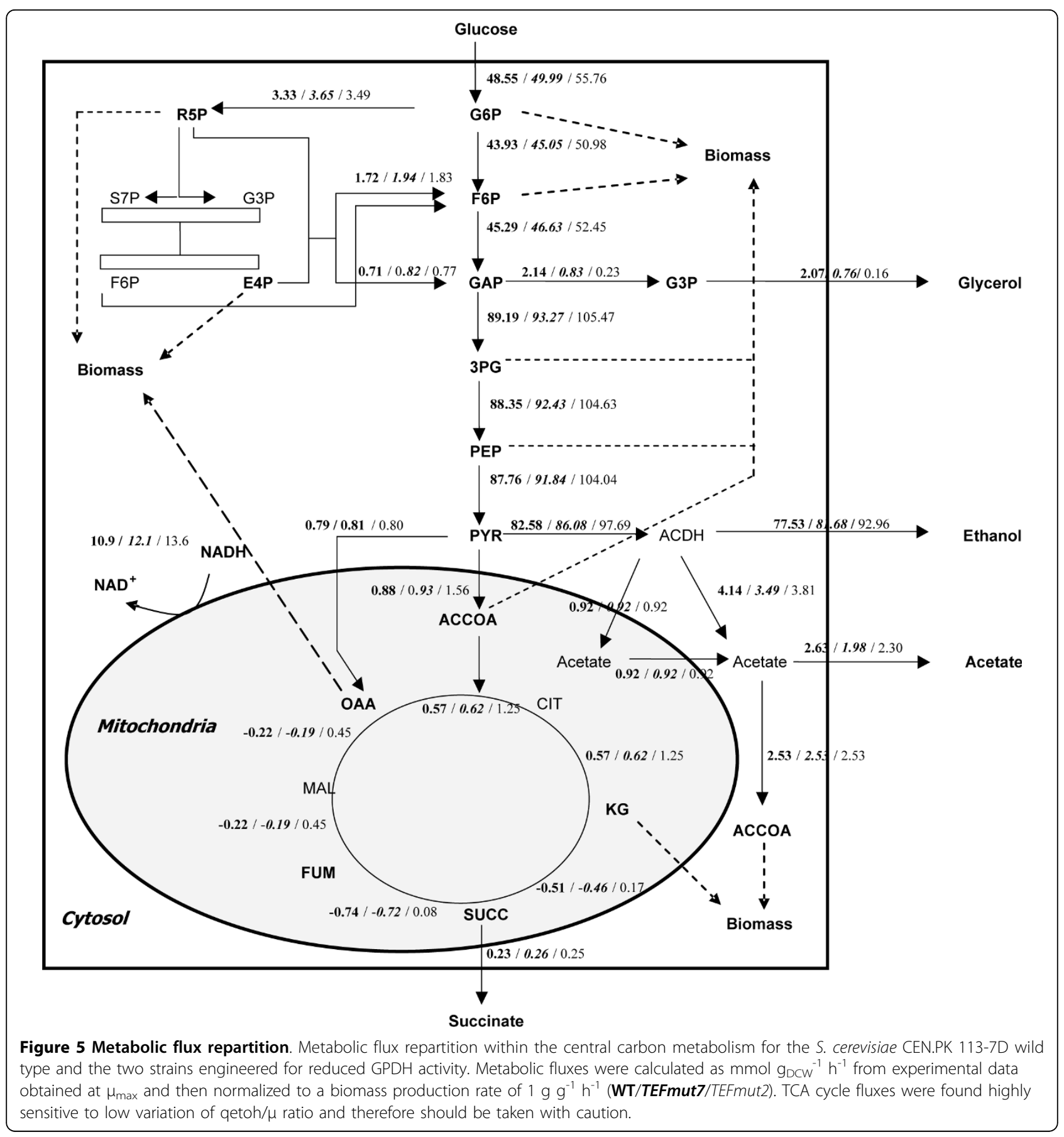

participation of ethanol biosynthesis and respiration to the NADH balance. The metabolic flux calculation revealed also an increased participation of the mitochondrial shuttles.

The mitochondrial shuttles were represented in the model by the glycerol-3-phosphate shuttle, but either an increased participation of the external NADH dehydrogenases $(N D E 1 / 2)$ or the glycerol-3-phosphate shuttle could take over the excess of cytosolic NADH $[8,48]$.
Therefore both systems likely participated to the transfer of cytosolic NADH into the mitochondria to be oxidised by the respiratory chain leading to the increase in the $\mathrm{O}_{2}$ consumption. However the reduction of the GPDH rate may have reduced the activity of the glycerol-3-phosphate shuttle compared to the NDEs in our engineered strains. As the glycerol-3-phosphate shuttle was reported to have a higher ATP/O ratio compared to the external NDEs, the reduction of the glycerol-3-phosphate shuttle activity 


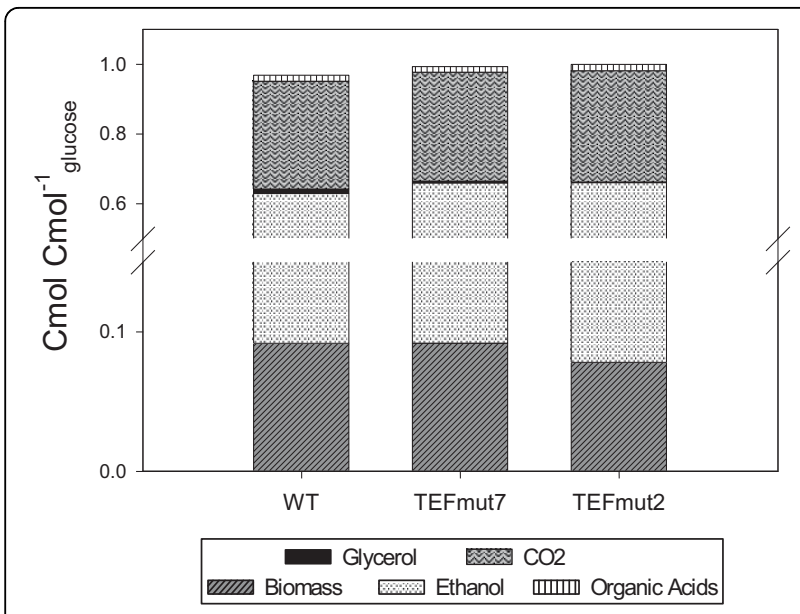

Figure 6 Carbon balances for the $S$. cerevisiae wild-type strain and the two mutants. Carbon balances and are expressed as Cmol ratio between the amount of metabolites produced (based on final masses at the end of fermentation) and glucose consumed.

could partly explain the reduction in Yx,ATP in our mutant strains [8].

One alternate explanation for growth rate reduction might be that the reduction in the glycerol phosphate dehydrogenase rate created a competition for the L-G3P between the anabolic requirement in this metabolite and glycerol synthesis within the cell. L-G3P produced by the reduction of DHAP is indeed either dephosphorylated into glycerol by the glycerol phosphate phosphatases (GPP) or enters into the phospholipid synthesis pathway via the phosphatidic acid (Figure 1). The rate of dephosphorylation of L-G3P being far higher than the rate of its conversion into phosphatidic acid, this latter reaction might limit the growth rate.

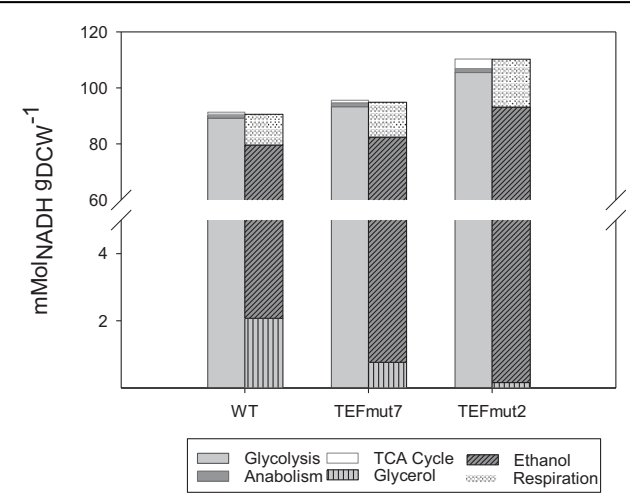

Figure $7 \mathrm{NADH}$ balances for the $S$. cerevisiae wild-type and the two mutants (TEFmut7 and TEFmut2). NADH balances were calculated for each strain at the time point when $\mu_{\max }$ was reached, respectively. Left bars correspond to generated NADH (by glycolysis, anabolism and TCA cycle); right bars correspond to the oxidized $\mathrm{NADH}$ (by glycerol and ethanol formation pathways and respiration).
No relevant reduction of the maximum specific growth rate was observed in single gpd $\Delta$ deletion mutants in previous studies in aerobic conditions [16]. However ethanol production capacities of these strains were studied under experimental conditions where the cells did not face high glucose and high ethanol concentration as encountered in VHEP conditions. Glycerol is known to play a role in stress resistance, such as osmotic stress [10,49], ethanol and temperature stress [13]. Glycerol is the major compatible solute accumulated in yeast to increase cell turgor pressure to face hyperosmotic stress. Modifying the glycerol synthesis pathway should alter the ability of the cell to regulate its turgor pressure. However authors have suggested through the surface stress theory that a fine tuned turgor pressure is necessary for yeast growth [50,51]. Thus an impact of the osmotic stress on the $\mu_{\max }$ of our strains under our conditions cannot be completely excluded.

Despite the impact on the growth rate and in clear contrast to the gpd1 $\operatorname{ggpd} 2 \Delta$ double deletion strain [20], ethanol tolerance was not affected by the genetic modifications carried out in the current study even though the fermentation conditions were exactly the same.

Finally we concluded that the two mutant strains were able to sustain a similar ethanol concentration than the wild type strain but with a higher energy expense. This higher energy demand increased the relative weight of energy production reactions over biomass synthesis in the metabolism of the mutants leading to an improved ethanol yield.

\section{Conclusions}

A prominent feature here is that fine-tuning the glycerol synthesis pathway (within the range 11-39\% of the wildtype capacity) allows the strains to keep their initial ethanol tolerance. Both engineered strains showed indeed a similar behaviour in terms of ethanol inhibition on growth quantified by a Pcritical/ $\mu$ value at $86 \pm 1 \mathrm{~g} \mathrm{~L}^{-1}$ and viability. Therefore we conclude that reducing the glycerol synthesis down to $11 \%$ of the wild type capacity did not affect the strain robustness in terms of ethanol tolerance, ethanol titer and productivity.

Additional file 1: S.cerevisiae metabolic network model. The Excel sheet "reactions" contains all the reactions in the model categorized by their metabolic pathway, the sheet "abbreviated metabolites" enlists the abbreviations of the metabolites, the sheet "symbolic variables" enlists the variables used for the description of macromolecule composition, the sheet "variables' values" shows for each variable the value used for the resolution of the equation system corresponding to the model.

\section{Abbreviations}

$a_{i}^{x}$ : Stoichiometric coefficient of metabolite $\mathrm{x}$ in reaction $\mathrm{i} ; q_{i}^{y}$ : Rate of reaction i belonging to metabolic pathway y; Ana: Anabolism; Gly: Glycolysis; OP: Oxidative Phosphorylation; TCA: Tricarboxylic acid cycle; Glyce: Glycerol; Etoh: Ethanol; NADHcon: NADH consumed; NADHpro: NADH produced. 


\section{Acknowledgements}

This work was supported by grants from the P2R Program (French Ministry of Foreign Affairs, CNRS and DAAD) and from the ANR program (ANR-05BIOE-007). J. Pagliardini gratefully acknowledges financial doctoral support by the ADEME and Danisco France.

\section{Author details}

'Université de Toulouse, INSA, UPS, INP, LISBP, 135 Av de Rangueil, F-31077 Toulouse France INRA, UMR792 Ingénierie des Systèmes Biologiques et des Procédés, F-31400 Toulouse, France CNRS, UMR5504, F-31400 Toulouse, France. ${ }^{2}$ Department of Molecular Microbiology, VIB, Kasteelpark Arenberg 31 - bus 2438, B-3001 Heverlee, Flanders, Belgium. ${ }^{3}$ Laboratory of Molecular Cell Biology, Institute of Botany and Microbiology, Katholieke Universiteit Leuven, Kasteelpark Arenberg 31 - bus 2438, B-3001 Heverlee, Flanders, Belgium. ${ }^{4}$ School of Engineering and Science, Jacobs University gGmbH, Campus Ring 1, 27579 Bremen, Germany.

\section{Authors' contributions}

JP, SG and CB contributed to the metabolic model set-up and flux calculation.

$\mathrm{GH}$ carried out the genetic work

$\mathrm{GH}, \mathrm{JP}, \mathrm{SG}$, and $\mathrm{CB}$ contributed to the fermentations experiments.

$\mathrm{SG}, \mathrm{EN}, \mathrm{CB}$, and $\mathrm{SA}$ conceived of the study, and participated in its design and coordination and helped to draft the manuscript.

All authors read and approved the final manuscript.

\section{Competing interests}

The authors declare that they have no competing interests.

Received: 17 December 2009 Accepted: 21 May 2010

Published: 21 May 2010

\section{References}

1. Eriksson P, André L, Ansell R, Blomberg A, Adler L: Cloning and characterization of GPD2, a second gene encoding sn-glycerol 3phosphate dehydrogenase $\left(\mathrm{NAD}^{+}\right)$in Saccharomyces cerevisiae, and its comparison with GPD1. Molecular Microbiology 1995, 17(1):95-107.

2. Gancedo C, Gancedo JM, Sols A: Glycerol Metabolism in Yeasts. European Journal of Biochemistry 1968, 5(2):165-172.

3. Bjorkqvist $\mathrm{S}$, Ansell R, Adler L, Liden G: Physiological response to anaerobicity of glycerol-3-phosphate dehydrogenase mutants of Saccharomyces cerevisiae. Applied Environmental Microbiology 1997, 63(1):128-132.

4. Oura E: Reaction products of yeast fermentations. Process Biochememistry 1977, 12:19-21

5. Albers E, Larsson C, Liden G, Niklasson C, Gustafsson L: Influence of the nitrogen source on Saccharomyces cerevisiae anaerobic growth and product formation. Applied Environmental Microbiology 1996, 62(9):3187-3195.

6. van Dijken J, Scheffers WA: Redox balances in the metabolism of sugars by yeasts. FEMS Microbiology Letters 1986, 32:199-224.

7. Ansell $R$, Granath $K$, Hohmann S, Thevelein JM, Adlerand L: The two isoenzymes for yeast $\mathrm{NAD}^{+}$-dependent glycerol 3-phosphate dehydrogenase encoded by GPD1 and GPD2 have distinct roles in osmoadaptation and redox regulation. The EMBO journal 1997. 16:2179-2187.

8. Rigoulet M, Aguilaniu H, Avéret N, Bunoust O, Camougrand N, GrandierVazeille X, Larsson C, Pahlman IL, Manon S, Gustafsson L: Organization and regulation of the cytosolic NADH metabolism in the yeast Saccharomyces cerevisiae. Molecular and Cellular Biochemistry 2004, 256-257(1):73.

9. Athenstaedt K, Daum G: Phosphatidic acid, a key intermediate in lipid metabolism. European Journal Biochemistry 1999, 266(1):1-16.

10. Hohmann S: Osmotic Stress Signaling and Osmoadaptation in Yeasts. Microbiology and Molecular Biology Review 2002, 66(2):300-372.

11. Blomberg A, Adler $L$ : Roles of glycerol and glycerol-3-phosphate dehydrogenase $\left(\mathrm{NAD}^{+}\right)$in acquired osmotolerance of Saccharomyces cerevisiae. Journal of Bacteriology 1989, 171(2):1087-1092.

12. Luyten K, Albertyn J, Skibbe WF, Prior BA, Ramos J, Thevelein JM, Hohmann S: Fps1, a yeast member of the MIP family of channel proteins, is a facilitator for glycerol uptake and efflux and is inactive under osmotic stress. The EMBO Journal 1995, 14:1360-1371.
13. Aldiguier AS, Alfenore S, Cameleyre X, Goma G, Uribelarrea JL, Guillouet SE, Molina-Jouve C: Synergistic temperature and ethanol effect on Saccharomyces cerevisiae dynamic behaviour in ethanol bio-fuel production. Bioprocess and Biosystems Engineering 2004, 26(4):217.

14. Michnick S, Roustan JL, Remize F, Barre P, Dequin S: Modulation of Glycerol and Ethanol Yields During Alcoholic Fermentation in Saccharomyces cerevisiae Strains Overexpressed or Disrupted for GPD1 Encoding Glycerol 3-Phosphate Dehydrogenase. Yeast 1997, 13(9):783-793.

15. Valadi H, Larsson C, Gustafsson L: Improved ethanol production by glycerol-3-phosphate dehydrogenase mutants of Saccharomyces cerevisiae. Applied Microbiology and Biotechnology 1998, 50(4):434.

16. Nissen TL, Hamann CW, Kielland-Brandt MC, Nielsen J, Villadsen J: Anaerobic and aerobic batch cultivations of Saccharomyces cerevisiae mutants impaired in glycerol synthesis. Yeast 2000, 16(5):463-474.

17. Guo Zp, Zhang L, Ding Zy, Wang ZX, Shi GY: Interruption of glycerol pathway in industrial alcoholic yeasts to improve the ethanol production. Applied Microbiology and Biotechnology 2009, 82(2):287.

18. Nissen TL, Kielland-Brandt MC, Nielsen J, Villadsen J: Optimization of Ethanol Production in Saccharomyces cerevisiae by Metabolic Engineering of the Ammonium Assimilation. Metabolic Engineering 2000, 2(1):69-77.

19. Bro C, Regenberg B, Förster J, Nielsen J: In silico aided metabolic engineering of Saccharomyces cerevisiae for improved bioethanol production. Metabolic Engineering 2006, 8(2):102.

20. Boulahya K: Evaluation des potentialités fermentaires de souches mutées de $S$. cerevisiae en vue d'une production nulle de glycérol dans une fermentation éthanolique. Toulouse: Université de Toulouse, INSA 2005.

21. Kong QX, Cao LM, Zhang AL, Chen X: Overexpressing GLT1 in gpd1 $\Delta$ mutant to improve the production of ethanol of Saccharomyces cerevisiae. Applied Microbiology and Biotechnology 2007, 73(6):1382.

22. Kong QX, Zhang AL, Cao LM, Chen X: Over-expressing GLT1 in a gpd2 $\Delta$ mutant of Saccharomyces cerevisiae to improve ethanol production. Applied Microbiology and Biotechnology 2007, 75(6):1361.

23. Zhang A, Kong Q, Cao L, Chen X: Effect of FPS1 deletion on the fermentation properties of Saccharomyces cerevisiae. Letters in Applied Microbiology 2007, 44(2):212-217.

24. Kong QX, Gu JG, Cao LM, Zhang AL, Chen X, Zhao XM: Improved production of ethanol by deleting FPS1 and over-expressing GLT1 in Saccharomyces cerevisiae. Biotechnology Letters 2006, 28(24):2033.

25. Cao L, Zhang A, Kong Q, Xu X, Josine TL, Chen X: Overexpression of GLT1 in fps1D gpdD mutant for optimum ethanol formation by Saccharomyces cerevisiae. Biomolecular Engineering 2007, 24(6):638

26. Alfenore S, Cameleyre X, Benbadis L, Bideaux C, Uribelarrea JL, Goma G, Molina-Jouve C, Guillouet SE: Aeration strategy: a need for very high ethanol performance in Saccharomyces cerevisiae fed-batch process. Applied Microbiology and Biotechnology 2004, 63(5):537.

27. Bideaux C, Alfenore S, Cameleyre X, Molina-Jouve C, Uribelarrea JL, Guillouet SE: Minimization of Glycerol Production during the HighPerformance Fed-Batch Ethanolic Fermentation Process in Saccharomyces cerevisiae, Using a Metabolic Model as a Prediction Tool. Applied Environmental Microbiology 2006, 72(3):2134-2140.

28. Alper H, Fischer C, Nevoigt E, Stephanopoulos G: Tuning genetic control through promoter engineering. PNAS 2005, 102(36):12678-12683.

29. Nevoigt E, Kohnke J, Fischer CR, Alper H, Stahl U, Stephanopoulos G: Engineering of Promoter Replacement Cassettes for Fine-Tuning of Gene Expression in Saccharomyces cerevisiae. Applied Environmental Microbiology 2006, 72(8):5266-5273.

30. Sambrook J, Maniatis T, Fritsch EF: Molecular cloning: a laboratory manual. Cold Spring Harbor, N.Y: Cold Spring Harbor Laboratory, 21989.

31. Alfenore S, Molina-Jouve C, Guillouet S, Uribelarrea JL, Goma G, Benbadis L: Improving ethanol production and viability of Saccharomyces cerevisiae by a vitamin feeding strategy during fed-batch process. Applied Microbiology and Biotechnology 2002, 60(1):67.

32. Gueldener U, Heinisch J, Koehler GJ, Voss D, Hegemann JH: A second set of loxP marker cassettes for Cre-mediated multiple gene knockouts in budding yeast. Nucleic Acids Res 2002, 30(6):e23.

33. Gietz RD, Schiestl RH: Applications of high efficiency lithium acetate transformation of intact yeast cells using single-stranded nucleic acids as carrier. Yeast 1991, 7(3):253-263.

34. Nevoigt E, Stahl U: Reduced pyruvate decarboxylase and increased glycerol-3-phosphate dehydrogenase $\left[\mathrm{NAD}^{+}\right]$levels enhance glycerol production in Saccharomyces cerevisiae. Yeast 1996, 12(13):1331-1337. 
35. Duboc P, Stockar Uv: Systematic errors in data evaluation due to ethanol stripping and water vaporization. Biotechnology and Bioengineering 1998, 58(4):428-439.

36. Bideaux C, Goma G, Uribelarrea UL, Dahhou B, Roux G: Stoichiometric modelling approach for microbial cultures monitoring. International Journal of Modelling, Identification and Control 2008, 3:413.

37. Ryan ED, Kohlhaw GB: Subcellular Localization of Isoleucine-Valine Biosynthetic Enzymes in Yeast. Jounal of Bacteriology 1974, 120(2):631-637.

38. Brandriss MC, Magasanik B: Subcellular compartmentation in control of converging pathways for proline and arginine metabolism in Saccharomyces cerevisiae. Journal of Bacteriology 1981, 145(3):1359-1364.

39. Kispal G, Steiner H, Court DA, Rolinski B, Lill R: Mitochondrial and Cytosolic Branched-chain Amino Acid Transaminases from Yeast, Homologs of the myc Oncogene-regulated Eca39 Protein. Journal of Biological Chemistry 1996, 271(40):24458-24464

40. Maaheimo H, Fiaux J, Cakar ZP, Bailey JE, Sauer U, Szyperski T: Central carbon metabolism of Saccharomyces cerevisiae explored by biosynthetic fractional $13 \mathrm{C}$ labeling of common amino acids. European Journal of Biochemistry 2001, 268(8):2464-2479.

41. Förster J, Famili I, Fu P, Palsson BÃ, Nielsen J: Genome-Scale Reconstruction of the Saccharomyces cerevisiae Metabolic Network. Genome Research 2003, 13(2):244-253.

42. Duarte NC, Herrgard MJ, Palsson BO: Reconstruction and Validation of Saccharomyces cerevisiae iND750, a Fully Compartmentalized GenomeScale Metabolic Model. Genome Research 2004, 14:1298-1309.

43. Gombert AK, Moreira dos Santos M, Christensen B, Nielsen J: Network Identification and Flux Quantification in the Central Metabolism of Saccharomyces cerevisiae under Different Conditions of Glucose Repression. Journam/ of Bacteriology 2001, 183(4):1441-1451.

44. Fiaux J, Cakar ZP, Sonderegger M, Wuthrich K, Szyperski T, Sauer U: Metabolic-Flux Profiling of the Yeasts Saccharomyces cerevisiae and Pichia stipitis. Eukaryotic Cell 2003, 2(1):170-180.

45. Remize F, Cambon B, Barnavon L, Dequin S: Glycerol formation during wine fermentation is mainly linked to Gpd1p and is only partially controlled by the HOG pathway. Yeast 2003, 20(15):1243-1253.

46. Olz R, Larsson K, Adler L, Gustafsson L: Energy flux and osmoregulation of Saccharomyces cerevisiae grown in chemostats under $\mathrm{NaCl}$ stress. Journal of Bacteriology 1993, 175(8):2205-2213.

47. Heyland J, Fu J, Blank LM: Correlation between TCA cycle flux and glucose uptake rate during respiro-fermentative growth of Saccharomyces cerevisiae. Microbiology 2009, mic.0.030213-030210.

48. Pahlman II, Larsson C, Averet N, Bunoust O, Boubekeur S, Gustafsson L, Rigoulet M: Kinetic Regulation of the Mitochondrial Glycerol-3-phosphate Dehydrogenase by the External NADH Dehydrogenase in Saccharomyces cerevisiae. Journal of Biological Chemistry 2002, 277(31):27991-27995.

49. Nevoigt $\mathrm{E}$, Stahl U: Osmoregulation and glycerol metabolism in the yeast Saccharomyces cerevisiae. FEMS Microbiology Reviews 1997, 21(3):231-241.

50. Slaughter B, Li R: Toward a molecular interpretation of the surface stress theory for yeast morphogenesis. Current Opinion in Cell Biology 2006, 18(1):47.

51. Koch $\mathrm{A}$ : The surface stress theory of microbial morphogenesis. Advances in Microbial Physiology 1983, 24:301-366.

52. van Dijken JP, Bauer J, Brambilla L, Duboc P, Francois JM, Gancedo C, Giuseppin MLF, Heijnen JJ, Hoare M, Lange HC, et al: An interlaboratory comparison of physiological and genetic properties of four Saccharomyces cerevisiae strains. Enzyme and Microbial Technology 2000, 26(9-10):706.

doi:10.1186/1475-2859-9-36

Cite this article as: Pagliardini et al:: Quantitative evaluation of yeast's requirement for glycerol formation in very high ethanol performance fed-batch process. Microbial Cell Factories 2010 9:36.

\section{Submit your next manuscript to BioMed Central and take full advantage of:}

- Convenient online submission

- Thorough peer review

- No space constraints or color figure charges

- Immediate publication on acceptance

- Inclusion in PubMed, CAS, Scopus and Google Scholar

- Research which is freely available for redistribution

Submit your manuscript at www.biomedcentral.com/submit
Ciomed Central 